\title{
Toward Web Information Integration on 3D Virtual Space
}

\author{
Yasuhiko Kitamura $^{1}$, Noriko Nagata ${ }^{1}$, Masataka Ueno $^{1}$, \\ and Makoto Nagamune ${ }^{2}$ \\ 1 Department of Informatics, Kwansei Gakuin University, Japan \\ \{ykitamura, nagata, mueno\}aksc.kwansei.ac.jp \\ ${ }^{2}$ Graduate School of Informatics, Kyoto University, Japan \\ nagamuneddb.soc.i.kyoto-u.ac.jp
}

\begin{abstract}
We report an implementation of GeneSys, which is a Web information integration system on 3D virtual space. We have built Kwansei Gakuin Kobe Sanda Campus as a virtual space called VKSC in which character agents navigate a user. VKSC reflects the Web information concerning weather, school calendar, and laboratories in the campus and the behaviour of agents changes depending on the information. Conventional Web systems mainly aim at providing information and knowledge to users, but GeneSys can additionally provide virtual experiences to users.
\end{abstract}

\section{Introduction}

Character agents [6], which are also called life-like characters [8], believable agents [1], or embodied conversational agents (ECA) [2], provide a natural and interactive interface between computers and human users by incorporating conversational communication technique in the interface. They become more attractive and entertaining by adding emotional aspects to them.

A number of commercial products have been developed by Microsoft1, Extempo[, Haptek ${ }^{3}$, Artificial Life 4 , and so on. These agents still need to be improved because of their limited communication skills, but have a potential to be an ultimate interface between computers and human users. For example, when we get in trouble to use a computer or a software, the easiest way to fix the problem is just to ask our colleague who knows the system well or to make a phone call to a support center of the system developer. Unfortunately, such colleague or center is often unavailable or busy, so we have to take an alternative way to consult Web sites that contain FAQs about the system, but actually we often have another problem to find a right answer in the Web sites. If a character agent is available on the Web sites and can answer our questions appropriately through dialogue on behalf a staff of the support center, it must be very helpful and appreciated.

\footnotetext{
${ }^{1}$ http://www.microsoft.com/msagent/

${ }^{2}$ http://www.extempo.com/

${ }^{3} \mathrm{http}: / /$ www.haptek.com/

${ }^{4}$ http://www.artificial-life.com/

F. Kishino et al. (Eds.): ICEC 2005, LNCS 3711, pp. 445455 2005.

(C) IFIP International Federation for Information Processing 2005
} 
We have been working on character agents as a Web interface [6/5/4]. Venus and Mars [4] is a cooking recipe search engine which employs multiple character agents. Each agent is an expert on a specific domain such as cooking recipes, health, and local specialities, respectively. These agents collaborate to answer a question such as "let me know a good recipe for recovering from cold. " This question can not be answered by a single agent because that requires both knowledge of cocking recipes and health. Recommendation Battlers [5] is a restaurant recommendation system employing two character agents. Each agent collects restaurant information from an individual Web site, and recommends restaurants to a user competing with another agent. These agents can be called presentation agents and related works have been done in DFKI [9].

In the previous applications, character agents are just attached to a Web browser, so they can move up, down, right, and left only and their actions are limited in the two dimensional space. In this paper, we extend the world where agents live from two dimensional to three dimensional space and propose a Web information integration scheme on the $3 \mathrm{D}$ virtual space.

\section{Web Information Integration on 3D Virtual Space}

In this paper, we provide a three dimensional virtual space which is tightly connected to the Web information concerning the virtual space. Character agents in the virtual space can have not only interactions through conversations with a user but also a more advanced form of interactions through taking actions in the space.

We can utilize a virtual space as a platform to integrate Web information which is distributed physically and logically. For example, in a university campus, there are many departments, and there are a number of laboratories in each department. Many of laboratories have their own Web pages, but the pages reside in an individual Web site and are maintained individually. Through a browser, it may be difficult to experience a fact that two laboratories are in the same campus. If we can integrate the Web pages related to the campus onto a 3D virtual space, it is easy for us to understand the geographical relation among the Web pages.

The 3D virtual space can be evolved by utilizing the information on the Web site. For example, by referring to the opening hours of a library that is available on the Web page, we can open and close the gate of the library on the 3D virtual space according to the time. This makes the virtual space more realistic.

Character agents take an important role as guides who navigate a user in the virtual space recommending the related Web information. For example, when the user wants to know the way from the main entrance of the campus to a laboratory which he/she wants to visit, an agent navigates the user in the virtual space. In addition, the agent recommends the related Web information, in which the user may show interest, encountered on the way to the destination.

Of course, the user can walk around the virtual space freely without character agents. He/she can get access to various information linked to the Web and can encounter various guide agents. This framework provides virtual experiences to users based on the Web information. 


\section{GeneSys}

We are developing a system called GeneSys (world Generating System) which is a prototype to embody Web information integration on a 3D virtual space. As shown in Figure 1 we classify elements in the virtual space into a background, objects, and agents. The background and objects are static elements which do not act autonomously by themselves. On the other hand, agents are active elements which act autonomously in the virtual space. Agents can influence objects but not the background. In other words, they can change the state of the objects but not that of the background.

As shown in Figure 2. Genesys integrates real world information such as Web information and the time onto a $3 \mathrm{D}$ virtual space. Web information is generally specified in HTML and a wrapper is used to extract information from a Web page. Genesys takes Web information and the time information as its input and produces descriptions of the background, objects, and agents as its output. These descriptions are displayed on FreeWalk3 [7] which is a tool to browse a 3D virtual space and character agents. Figure 3

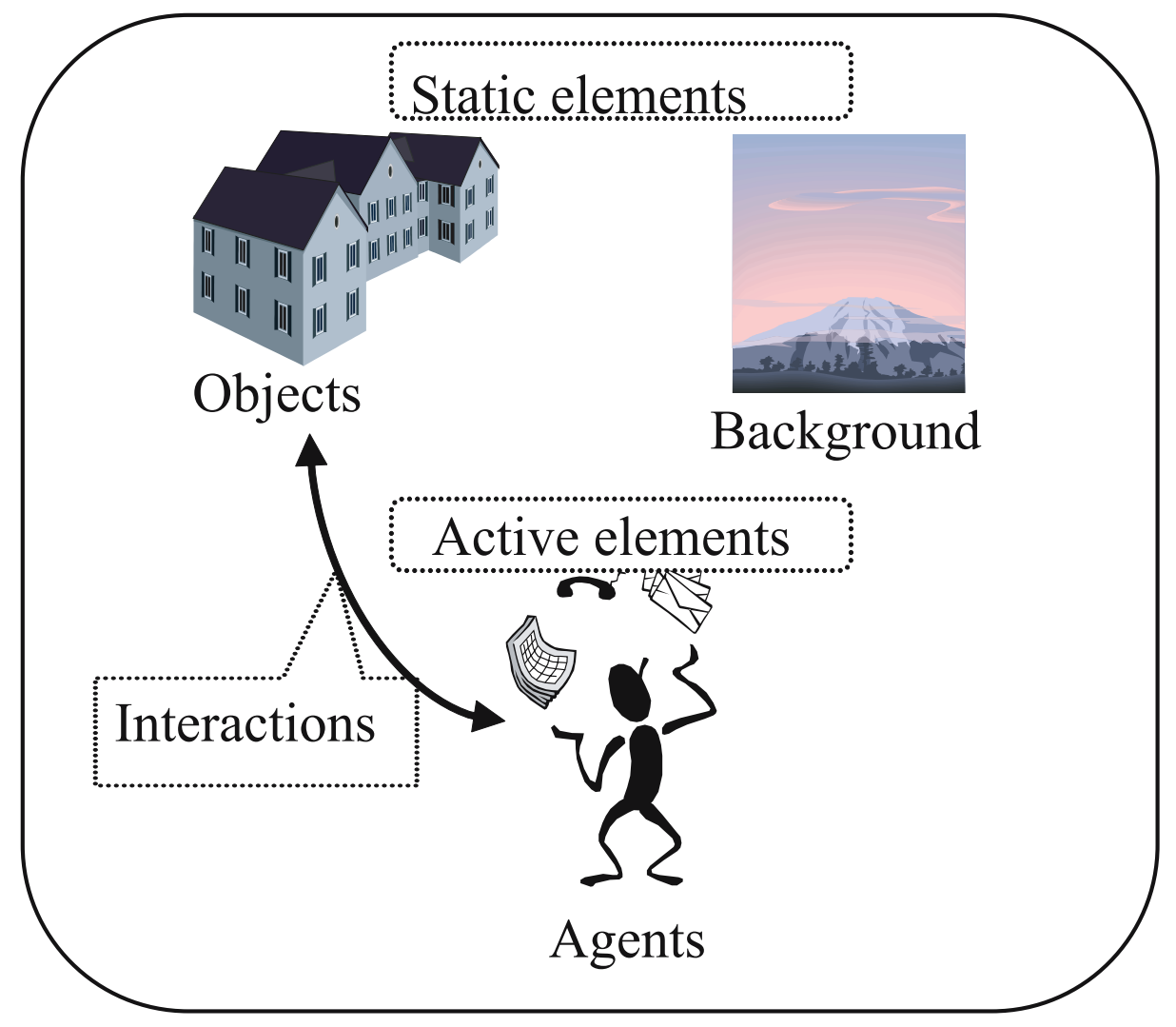

Fig. 1. Elements in 3D virtual space 


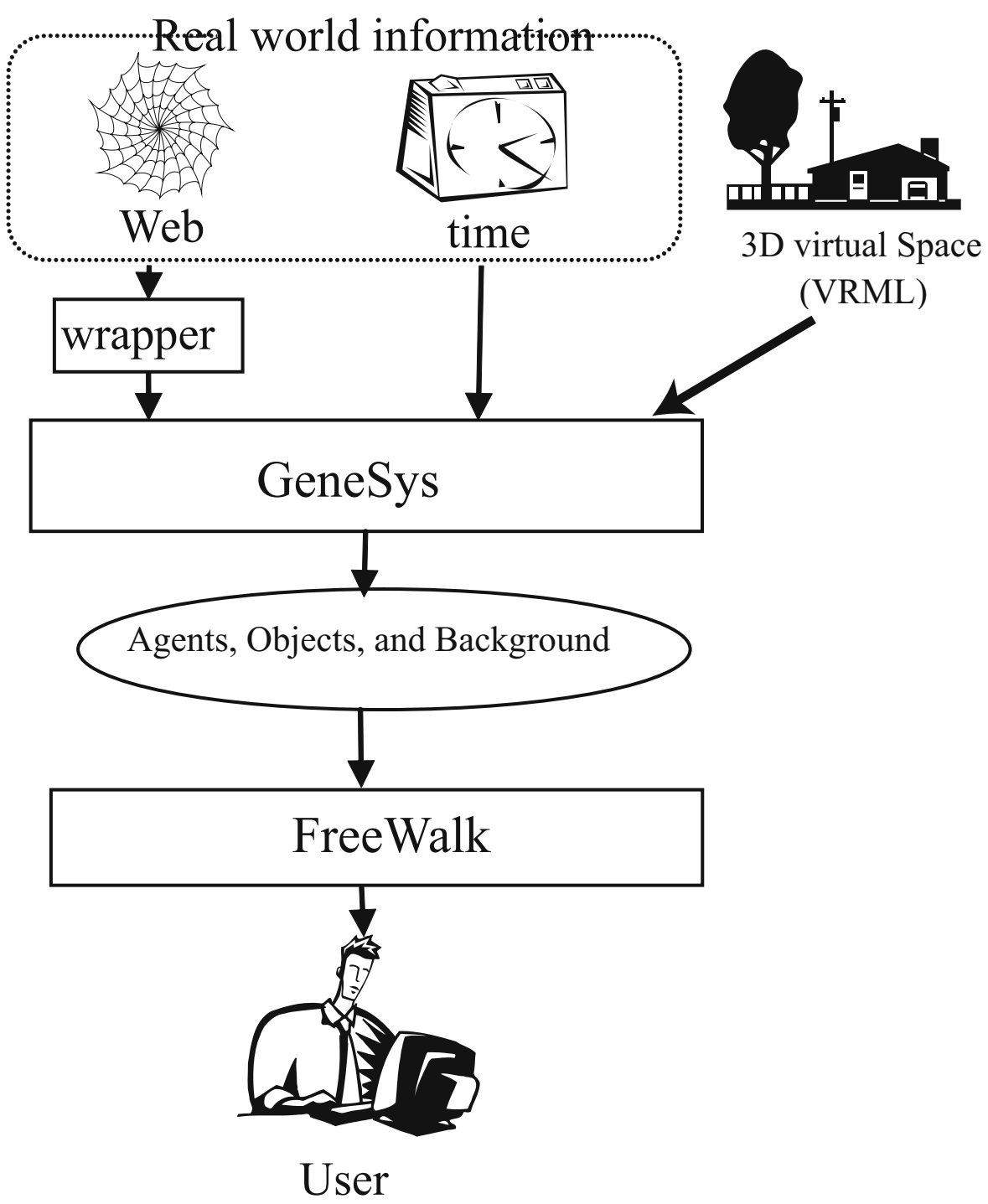

Fig. 2. Information integration on Genesys

shows an example of virtual space, consisting a virtual campus (Kobe Sanda Campus of Kwansei Gakuin University) and multiple character agents, displayed on FreeWalk3.

A related work on integrating Web information on a 3D virtual space has been done by Toru Ishida and his colleagues in the Digital City project [3]. A virtual shopping mall has been developed and shops in the mall are linked to their corresponding Web sites. If a user enters a shop, its Web page is opened in a browser to lead the user to its online shopping. The virtual space in the Digital City project is static and does not change. 
On the other hand, our virtual space generated by GeneSys is dynamic and changes depending on the Web information.

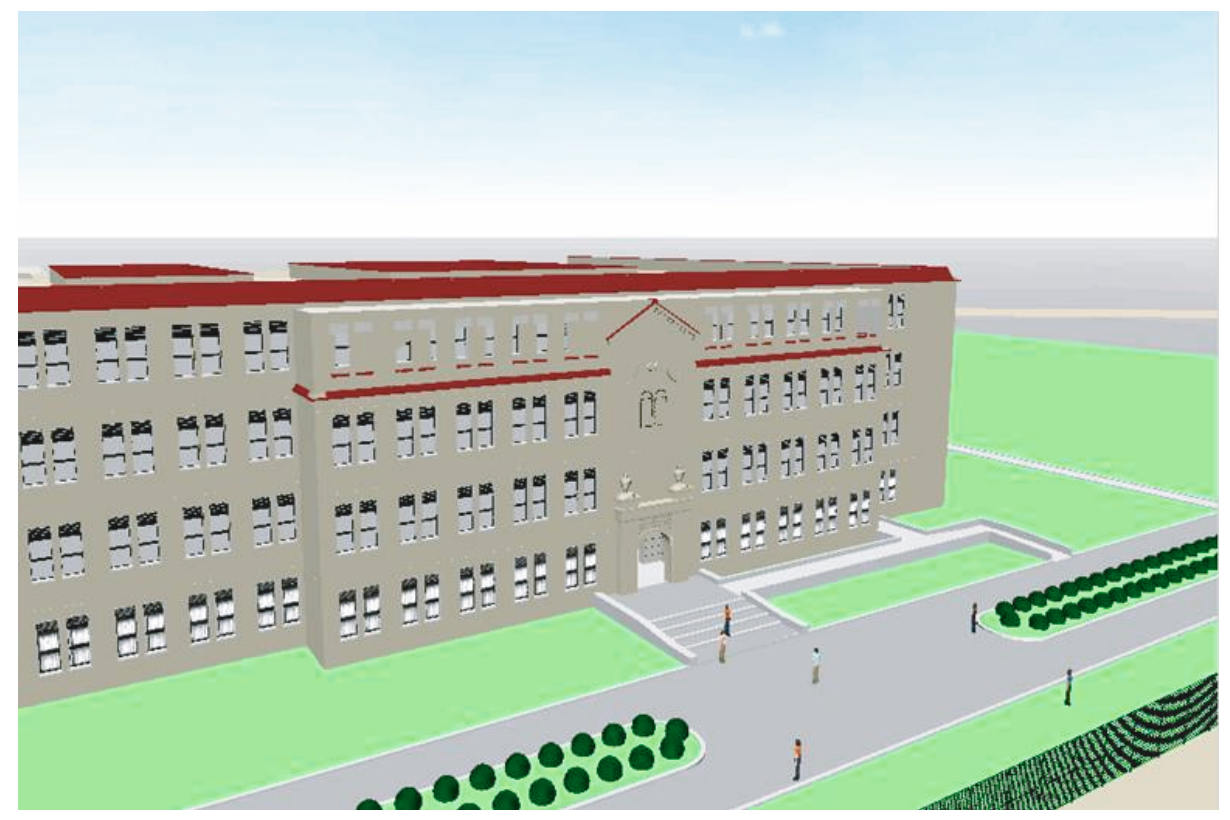

Fig. 3. Virtual campus and character agents on FreeWalk3

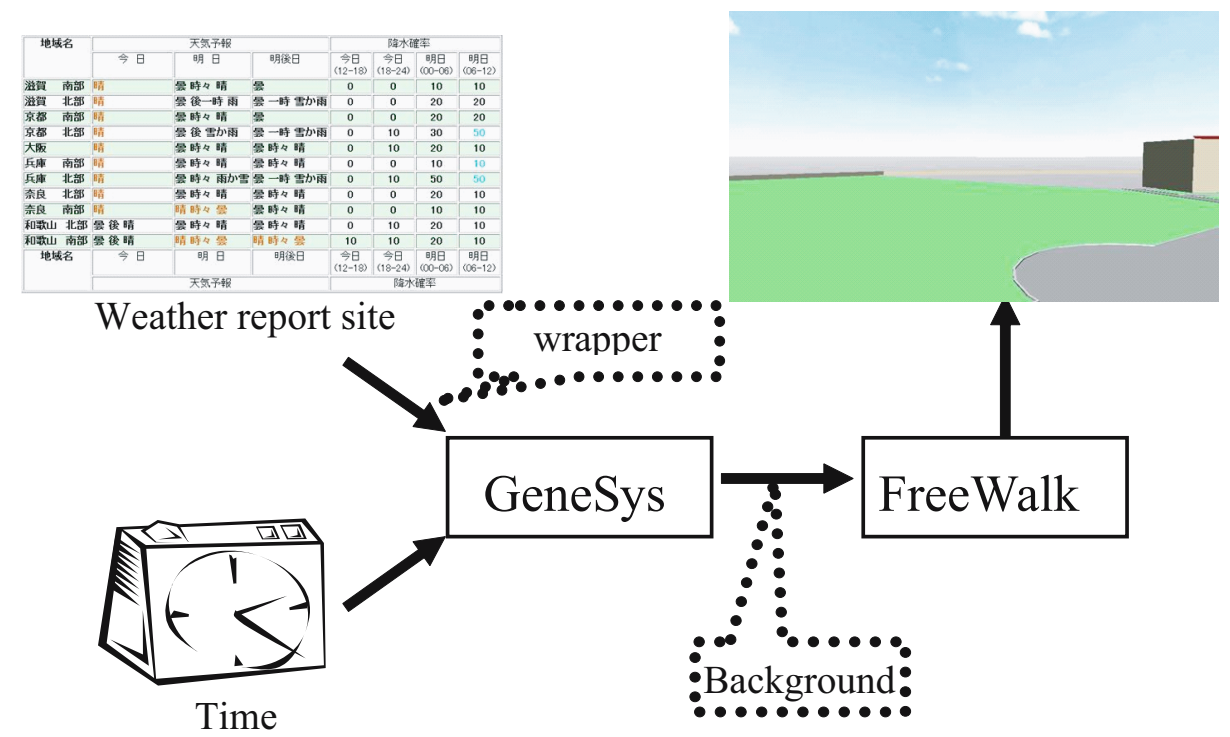

Fig. 4. Linking the Web and the background 


\section{Web Information Integration on GeneSys}

We describe how real world information obtained from the Web integrates onto a 3D virtual space by using an example of VKSC (Virtual Kobe Sanda Campus).

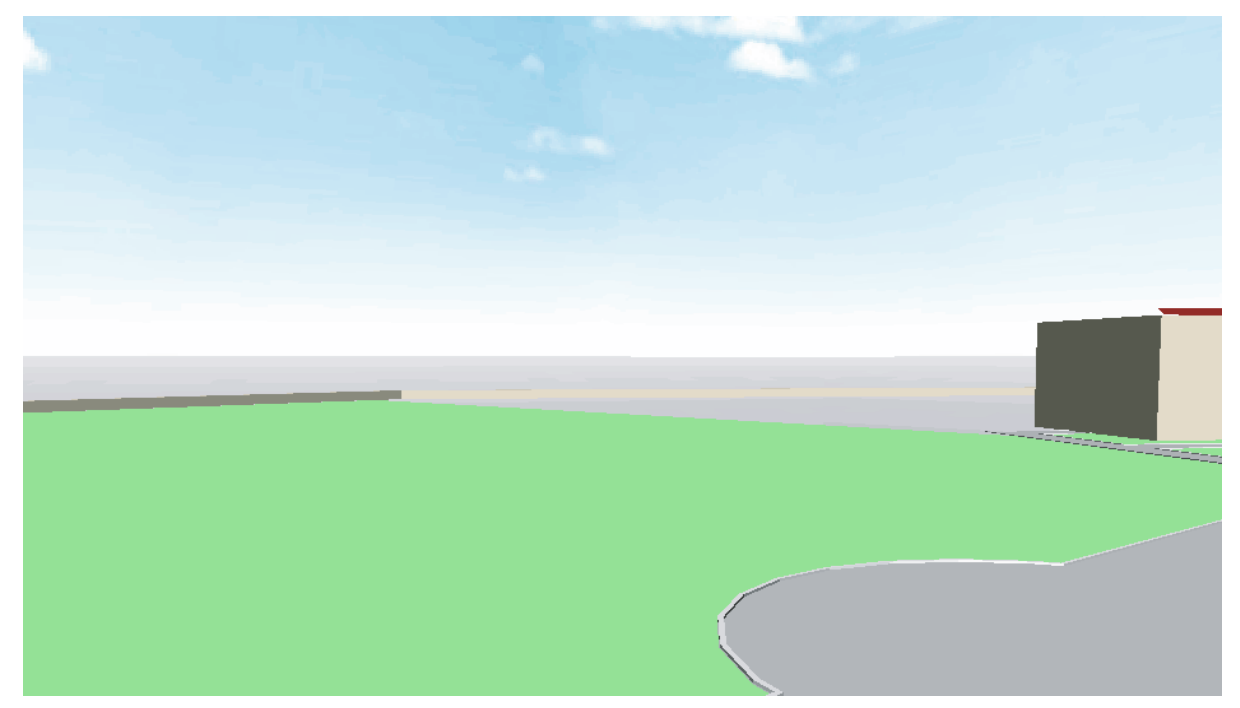

\section{(a) the day time}

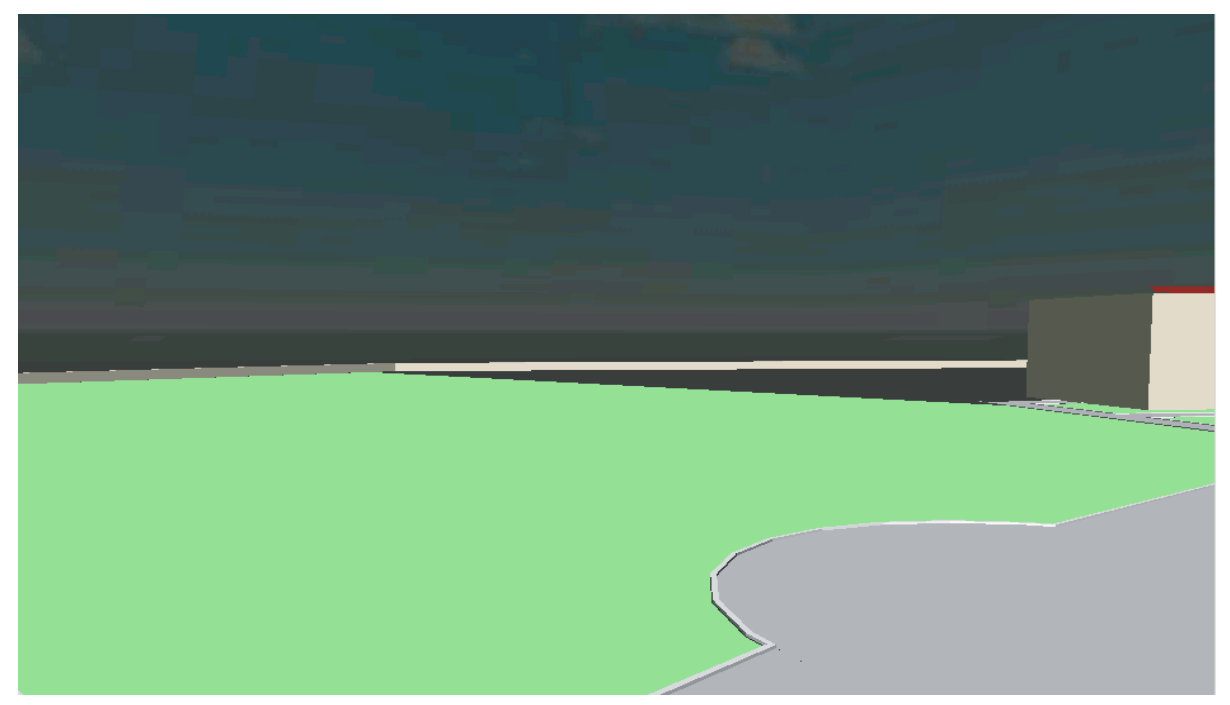

\section{(b) the night time}

Fig. 5. Backgrounds in day time and night time 


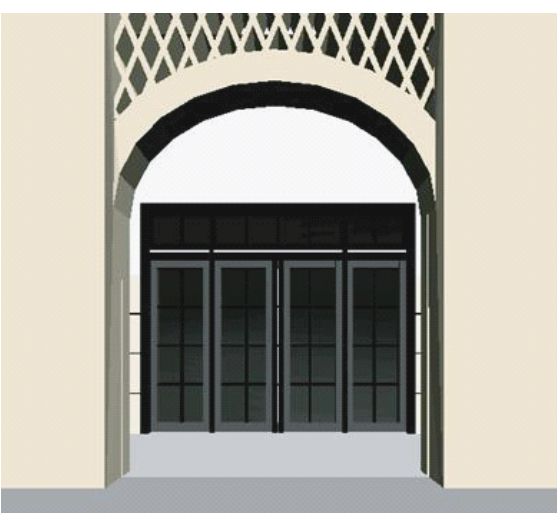

(a) gate closed

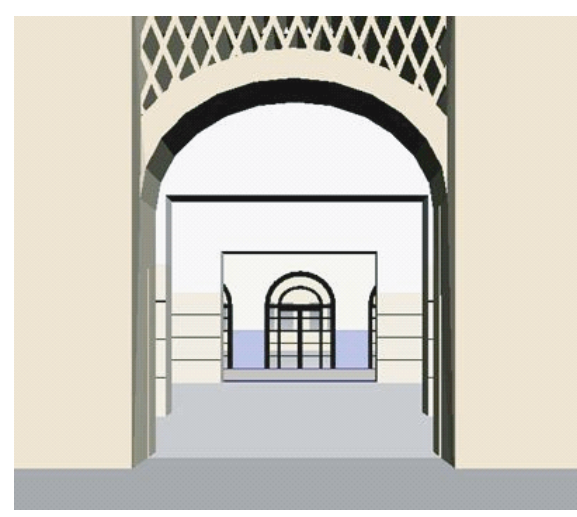

(b) gate open

Fig. 6. Linking the Web and objects

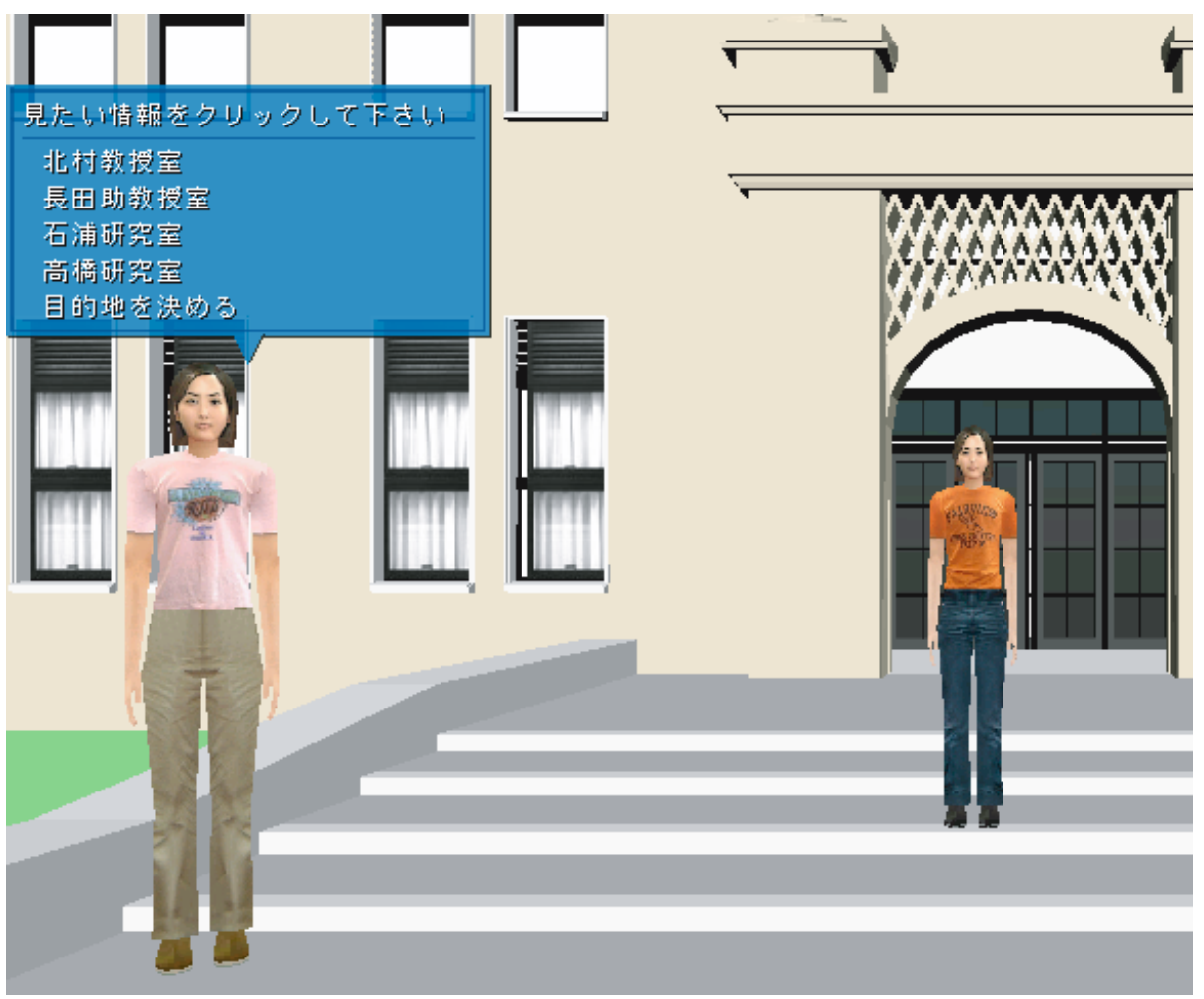

Fig. 7. A guide agent shows the list of laboratories 


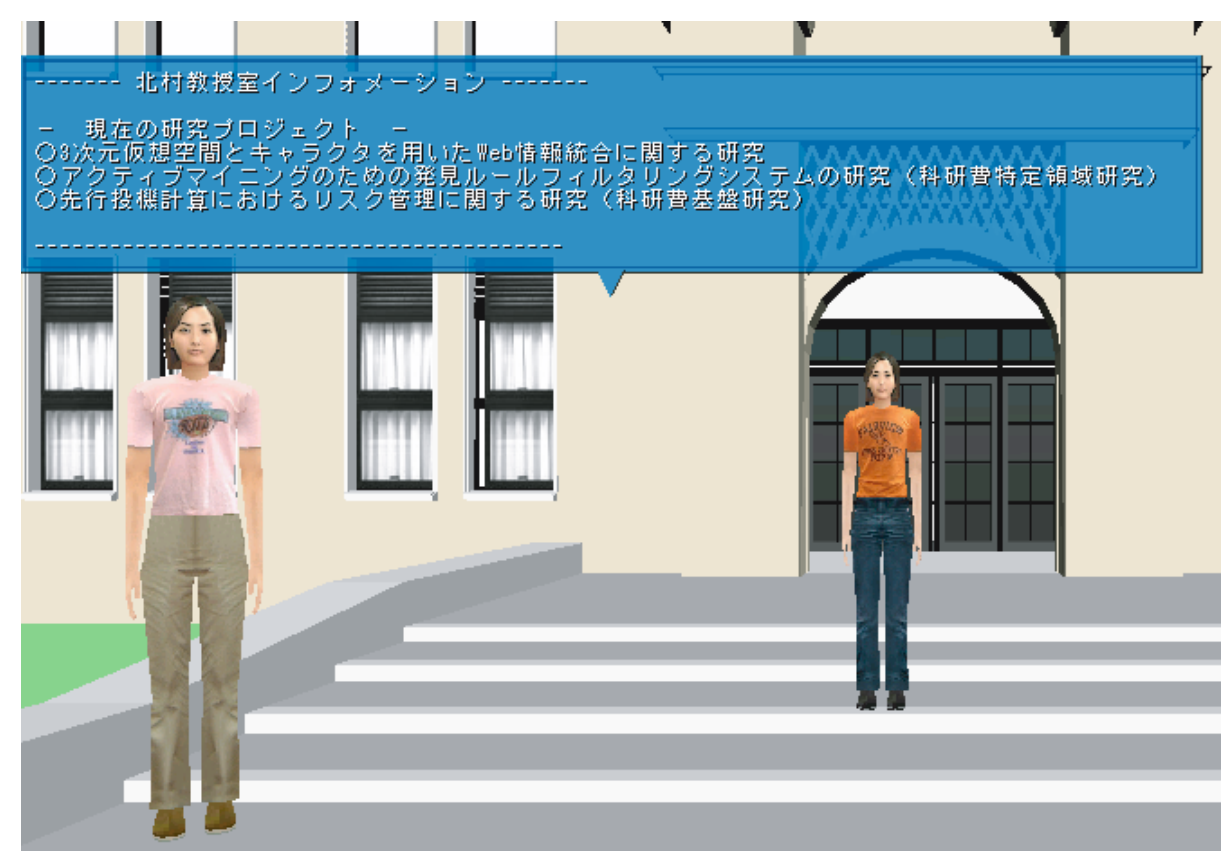

Fig. 8. A guide agent shows the information of laboratory

\subsection{Linking the Web and the Background}

As shown in Figure 4, Genesys collects the weather information of the southern Hyogo prefecture, where Kobe Sanda campus is located, from a weather report site 5 through a wrapper program. It also obtains the current time from the system clock. It then produces the background in which the weather reflects the Web information and the brightness reflects the current time. We show the difference between day and night of a fine day in Figure 4. Linking the Web and the time information to the background enhances the reality of the virtual space.

\subsection{Linking the Web and Objects}

GeneSys can make the calendar information in the university's Web sit@6 reflect the state of the main entrance in VKSC. When it is on holidays, the entrance is closed as shown in Figure 6 a) and when not, it is open as shown in Figure 6(b). Changes of the state of objects influence behaviours of character agents as mentioned later.

\subsection{Linking the Web and Agents}

The guide agents in VKSC navigate the user from the main entrance to a laboratory that the user wants to visit. At first, a guide in pink shows the list of laboratories (Figure 7).

\footnotetext{
${ }^{5}$ http://www.imoc.co.jp/yohou/yohou_4.htm

${ }^{6} \mathrm{http}: / /$ www.kwansei.ac.jp/student/years_calender.html
} 


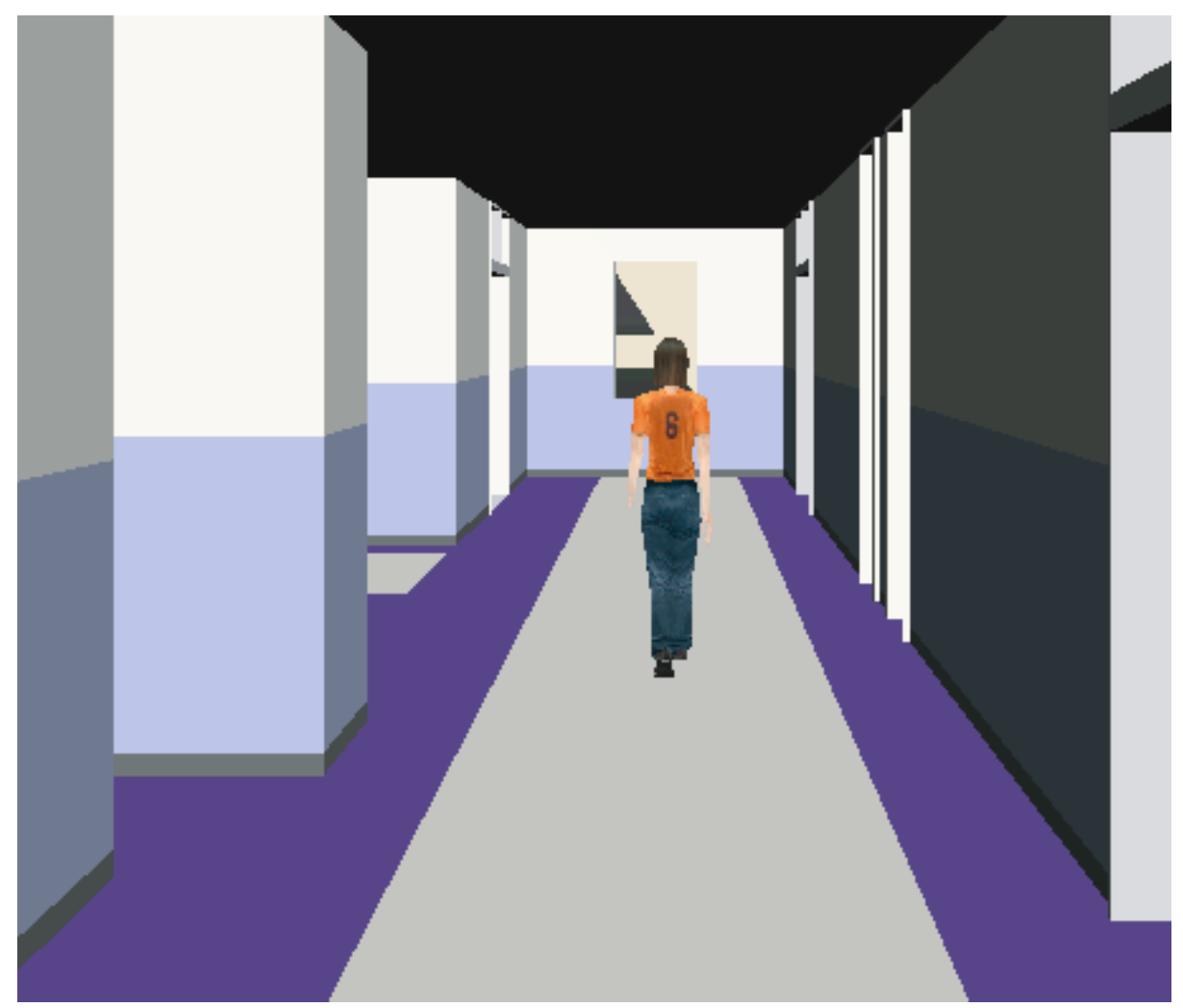

Fig. 9. A guide agent navigates the user in VKSC

It then shows the information of the laboratory chosen by the user. (Figure 8). Finally, another guide agent in orange navigates the user from the main entrance to the laboratory chosen (Figure 9).

We can send instructions to agents through the laboratory's Web pages. For example, we insert the following description into the Web page.

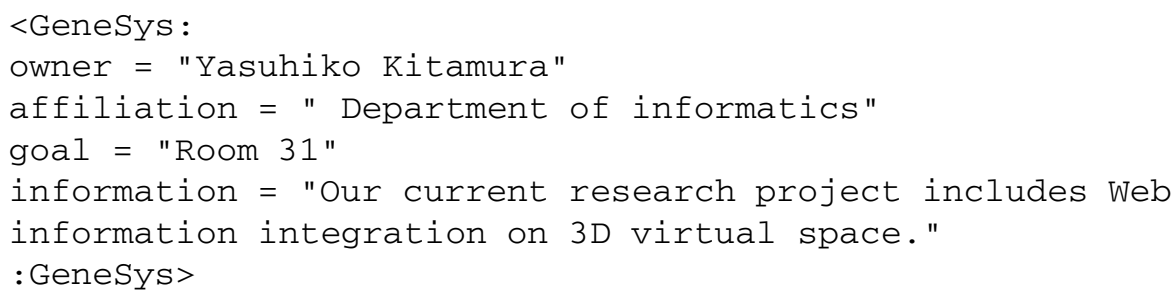

In this description, owner specifies the owner of the Web page, affiliation specifies the affiliation of the owner, goal specifies the destination to which a guide agent navigate a user, and information specifies the laboratory information that the 
guide agents answer when asked by a user. The guide agents collect this description through a wrapper and utilize it for their navigation.

When a laboratory is chosen by the user, the guide agents show the attribute information and navigate the user to the place specified by the goal attribute.

We can easily update the description in the Web page and the agents change their behaviour depending on the change.

\subsection{Interaction Between Objects and Agents}

The guide agents take actions according to the state of objects. For example, when the main entrance is closed on holidays, the agents bypass the main entrance and enter through a sub entrance which is open even on holidays. To embody this function, a simple planner based on the shortest path algorithm is embedded in the guide agents.

\section{Summary and Future Work}

This paper proposes a framework where Web information distributed on the Internet is integrated on a 3D virtual space and introduces a prototype called GeneSys. Genesys collects Web information related to the virtual space and produces the background, objects, and agents depending on the Web information. By using this scheme, we cab enhance the virtual space to be more realistic and dynamic according to the Web information.

The main goal of conventional Web systems is to provide information or knowledge to users. By integrating the Web information onto a 3D virtual space, it also provides a field where a user virtually experiences the information. Character agents take an important role in the virtual space to provide the information and to guide in the virtual space through natural and various ways of interactions with the user.

At present, a prototype has been just developed. From a viewpoint of character agents, our future work is to enhance the functionality of the agents. By using GeneSys, the $3 \mathrm{D}$ virtual space is updated according to the Web information, so the agents need to be adaptive to the updates. At present, they have just a simple form of planning scheme to find a shortest path to a destination, but they need to plan more elaborate aspects of agents such as conversation, gesture, actions, and emotion, to guide users more efficiently and naturally.

\section{References}

1. Joseph Bates. The Role of Emotion in Believable Agents, Communication of the ACM, 37(7):122-126, 1994.

2. Justine Cassell, Joseph Sullivann, Scott Prevost, and Elizabeth Churchill (Eds.), Embodied Conversational Agents, MIT Press, 2000.

3. Toru Ishida. Digital City Kyoto: Social Information Infrastructure for Everyday Life, Communications of the ACM, 45(7):76-81, 2002.

4. Yasuhiko Kitamura, Teruhiro Yamada, Takashi Kokubo, Yasuhiro Mawarimichi, Taizo Yamamoto, Toru Ishida. Interactive Integration of Information Agents on the Web, Matthias Klusch, Franco Zambonelli (Eds.), Cooperative Information Agents V, Lecture Notes in Artificial Intelligence 2182, Berlin et al.: Springer-Verlag, 1-13, 2001. 
5. Yasuhiko Kitamura, Toshiki Sakamoto, and Shoji Tatsumi. A Competitive Information Recommendation System and Its Behavior, Matthias Klusch, Sascha Ossowski, and Onn Shehory (Eds.), Cooperative Information Agents VI, Lecture Notes in Artificial Intelligence 2446, Berlin et al.: Springer-Verlag, 138-151, 2002.

6. Yasuhiko Kitamura. Web Information Integration Using Multiple Character Agents, Helmut Prendinger and Mitsuru Ishizuka (Eds.), Life-Like Characters: Tools, Affective Functions, and Applications, Springer-Verlag, 295-316, 2004.

7. Hideyuki Nakanishi. FreeWalk: A Social Interaction Platform for Group Behavior in a Virtual Space, International Journal of Human Computer Studies, 60(4):421-454, 2004.

8. Helmut Prendinger and Mitsuru Ishizuka (Eds.) Life-Like Characters: Tools, Affective Functions, and Applications, Springer-Verlag, 2004.

9. Thomas Rist, Elisabeth Andre, Stephan Baldes, Patrick Gebhard, Martin Klessen, Michael Kipp, Peter Rist, and Markus Scmitt. A Review of the Development of Embodied Presentation Agents and Their Application Fields, Helmut Prendinger and Mitsuru Ishizuka (Eds.), Life-Like Characters: Tools, Affective Functions, and Applications, Springer-Verlag, 295-316, 2004. 